\title{
EJNMMI Research: a new journal in nuclear medicine
}

\author{
Angelika Bischof Delaloye
}

Published online: 23 November 2010

(C) Springer-Verlag 2010

With the beginning of 2011 Springer has launched, after consultation with Ignasi Carrió, Editor-in-Chief of the European Journal of Nuclear Medicine and Molecular Imaging (EJNMMI), a companion journal, EJNMMI Research. With the increasing number of manuscripts that cannot be published by the EJNMMI because of reasons other than insufficient quality - space, redundancy, target audience etc. - it became obvious that there was a need for another forum where high-quality nuclear medicine research features could be communicated [1]. It was also clear that Open Access (OA) was the key to a rapid and wide dissemination of scientific results. A great number of funding organisations all over the world already require or at least encourage grant recipients to make their results available according to the OA paradigm. The costs of publication are lower than in traditional journals; and they are supported by the authors or their institutions rather than by the readers via subscription. The number of institutions across Europe and elsewhere that are members or supporting members of OA publishers, and consequently support the publication costs, is constantly growing.

EJNMMI Research will publish basic, translational and clinical research in nuclear medicine and related fields. It is conceived as a platform for rapid communication and lively debate. Therefore, besides original manuscripts and indepth reviews, interesting preliminary results should be submitted. In the same spirit, short communications and selected reports on unusual or rare conditions of general

\footnotetext{
A. Bischof Delaloye $(\square)$

Service of Nuclear Medicine,

University Hospital of Lausanne CHUV,

Rue du Bugnon 46,

1011, Lausanne, Switzerland

e-mail: Angelika.BischofDelaloye@chuv.ch
}

interest will be published. Editorials and letters to the editor should foster scientific exchange. Finally, educational material that deepens and enlarges understanding and knowledge in fields of high scientific interest is also welcome.

All submissions will be peer reviewed using criteria of scientific quality equivalent to those for the EJNMMI and comparable international journals. After acceptance, manuscripts will be rapidly available through the journal website (www.springer.com/13550) at Springer or at BioMed Central (www.ejnmmires.com). Manuscripts will immediately be indexed by PubMed Central as well as other web services such as Google, etc. This fast and broad availability is the great advantage of OA journals. Who has never been frustrated by refusal of access to a potentially interesting publication because his/her institution has no subscription to a given journal or publisher? Usually one would not cite a publication without studying the full text, as this is the only way to appreciate the quality and reliability of the results. OA gives immediate access to the full text of any publication to anybody who has an Internet connection; it is obvious that this overall availability of the complete data will favour citation.

BioMed Central currently publishes over 200 peerreviewed journals. More than half of them are indexed by Thomson Reuters (ISI), and many have already achieved remarkable impact factors. It is our ambition to guide EJNMMI Research into the club of renowned international journals. However, we need the contribution of all scientists working in the field of nuclear medicine and related sciences. We rely both on authors who are confident in this new approach to publication and dissemination of scientific results and knowledge and on reviewers who ensure that the appropriate quality standards are met. 
The new journal should not be understood as a competitor for the EJNMMI. The two journals share the same goal, to publish interesting and outstanding research data in the field of targeted imaging and therapy in basic, translational and clinical sciences. The means used to achieve this goal are different. There will inevitably be some overlap but we hope to keep it as small as possible. We are currently putting in place a mechanism that invites authors of papers that cannot be considered for publication in the EJNMMI to submit their work to EJNMMI Research. This should not be understood to imply that EJNMMI Research is a second-choice journal. Among the $81 \%$ of original papers rejected by the EJNMMI, many merit publication but cannot be accommodated by the EJNMMI mainly because of lack of space. I am grateful to Ignasi Carrió for his contributions in creating and shaping this new journal, in particular for agreeing to further accompany EJNMMI Research in the position of Associate Editor. As for myself, I will continue to function as Associate Editor of the EJNMMI. I am confident that this dual affiliation will allow us to closely follow the evolution of both journals and make the right decisions at the right time. For obvious reasons, a great number of the members of the Editorial Board of the EJNMMI Research are also on the EJNMMI Editorial Board. I take this opportunity to thank all the members of the new Editorial Board for having accepted this additional task, knowing that all of them already have more than enough activities on their plate. I especially thank them for their comments, spanning the entire spectrum from benevolent to enthusiastic.

Finally, EJNMMI Research will not be linked to the European Association of Nuclear Medicine and consequently will not publish guidelines or society news. We will, however, be happy to consider the publication of papers resulting from studies performed under the guidance of EANM Committees or the EARL (EANM Research Ltd).

We are very much looking forward to joining you all in the introduction and distribution of this new form of scientific communication in the field of nuclear medicine. I apologise in advance for any inconsistencies, delays or other problems that might arise at the beginning. We all know that any achievement depends on eagerness to learn, constancy of effort, and critical evaluation but also on humility - and a little bit of luck - from the learning phase onward. We will take care of the first four items in this list and trust in the fifth!

We look forward to receiving your manuscripts at www. springer.com/13550 or www.ejnmmires.com

\section{Reference}

1. Carrió I. Expanding the scope of EJNMMI in the era of electronic publishing. Eur J Nucl Med Mol Imaging. 2010;37:2229-30. doi:10.1007/s00259-010-1659-y. 